\title{
Hadronic weak decays of the charmed baryon $\Omega_{c}$
}

\author{
Shiyong Hu, Guanbao Meng, and Fanrong Xu๑* \\ Department of Physics, Jinan University, Guangzhou 510632, People's Republic of China
}

(Received 19 March 2020; accepted 18 May 2020; published 27 May 2020)

\begin{abstract}
Two-body hadronic weak decays of the charmed baryon $\Omega_{c}$, including the Cabibbo-favored (CF), singly Cabibbo-suppressed (SCS), and doubly Cabibbo-suppressed (DCS) modes, are studied systematically in this work. To estimate nonfactorizable contributions, we work in the pole model for the $P$-wave amplitudes and current algebra for the $S$-wave amplitudes. Among all the channels decaying into a baryon octet and a pseudoscalar meson, $\Omega_{c} \rightarrow \Xi^{0} \bar{K}^{0}$ is the only allowed CF mode. The predicted branching fraction of order $3.8 \%$ and large and positive decay asymmetry of order 0.50 indicate that a measurement of this mode in the near future is promising. Proceeding through purely nonfactorizable contributions, the SCS mode $\Omega_{c} \rightarrow \Lambda^{0} \bar{K}^{0}$ and DCS mode $\Omega_{c} \rightarrow \Lambda^{0} \eta$ are predicted to have branching fractions as large as $0.8 \%$ and $0.4 \%$, respectively. The two DCS modes $\Omega_{c} \rightarrow \Sigma^{0} \eta$ and $\Omega_{c} \rightarrow \Lambda^{0} \pi^{0}$ are suggested to serve as new physics searching channels for their vanishing SM background.
\end{abstract}

DOI: 10.1103/PhysRevD.101.094033

\section{INTRODUCTION}

The $\Omega_{c}$ baryon comprises a combination of a charm quark and two strange quarks. Classified by the SU(3) flavor symmetry, the $\Omega_{c}$ is one of the sextet charmed baryons. It is also the heaviest one with a mass [1],

$$
m=(2695.2 \pm 1.7) \mathrm{MeV},
$$

averaged over Belle [2], CLEO [3], and E687 [4] experiments.

Recently, there have been some experimental progresses on the study of $\Omega_{c}$. In 2017, LHCb observed five new narrow states of $\Omega_{c}$ via the decay channel $\Xi_{c}^{+} K^{-}$, while the light charmed baryon $\Xi_{c}^{+}$was reconstructed in the mode $p K^{-} \pi^{+}$[5]. Later in the same year, four of the five resonances were confirmed independently by Belle [6]. Then, various theoretical models have been proposed to interpret these new resonances, including excited states of the $\Omega_{c}$ baryon [7,8], pentaquark picture [9,10], and so on. The understanding of these $\Omega_{c}$ resonances is still an open question. Progress has also been made in the ground state $\Omega_{c}$. In 2018, LHCb reported a measurement of the $\Omega_{c}$ lifetime [11],

$$
\tau=(2.68 \pm 0.26) \times 10^{-13} \mathrm{~s},
$$

\footnotetext{
*fanrongxu@jnu.edu.cn
}

Published by the American Physical Society under the terms of the Creative Commons Attribution 4.0 International license. Further distribution of this work must maintain attribution to the author(s) and the published article's title, journal citation, and DOI. Funded by SCOAP ${ }^{3}$. which is nearly 4 times larger than the previous worldaverage value $(69 \pm 12)$ fs [12-14] and is consistent with a recent theoretical prediction [15].

It is well known that the decay of $\Omega_{c}$ is through weak interactions, which is distinct from other sextet charmed baryons as well as $\Omega_{c}$ excited states. However, about its weak decay information, we know much less than antitriplet charmed baryons $\left(\Lambda_{c}^{+}, \Xi_{c}^{0}, \Xi_{c}^{+}\right)$due to its low production. Though no absolute branching fraction of $\Omega_{c}$ has been measured so far, a ratio between the modes decaying into $\Xi^{0} \bar{K}^{0}$ and $\Omega^{-} \pi^{+}$,

$$
\frac{\mathcal{B}\left(\Omega_{c}^{0} \rightarrow \Xi^{0} \bar{K}^{0}\right)}{\mathcal{B}\left(\Omega_{c}^{0} \rightarrow \Omega^{-} \pi^{+}\right)}=1.64 \pm 0.26 \pm 0.12
$$

is reported by Belle using $980 \mathrm{fb}^{-1}$ of $e^{+} e^{-}$annihilation data [16], while the latter one is taken as a benchmark channel. As for the channels containing $\Omega^{-}$in final states, the semileptonic decay $\Omega_{c}^{-} \rightarrow \Omega^{-} e^{+} \nu_{e}$ was firstly observed by CLEO in 2012 [17]. With data accumulated both in Belle-II and LHCb, more interesting experimental results are anticipated. Hence, a theoretical study on weak decays of $\Omega_{c}$ is necessary and timely.

The theoretical studies of hadronic weak decays of the $\Omega_{c}$ baryon had a long history for several decades and a fast development in the 1990s [18-21]. It has been widely accepted that nonfactorizable contributions to decay amplitudes play an important role in the hadronic decays. Various methodologies were developed to describe the nonfactorizable contributions in charmed baryon decays, including the covariant confined quark model $[18,21]$, the pole model [20], and the pole model associated with current 
algebra [20]. Some new efforts have also been made in recent years $[22,23]$. In this work, we shall focus on the $1 / 2^{+} \rightarrow 1 / 2^{+}+0^{-}$decays of $\Omega_{c}$.

In the pole model, nonfactorizable $S$ - and $P$-wave amplitudes for $1 / 2^{+} \rightarrow 1 / 2^{+}+0^{-}$decays are dominated by $1 / 2^{-}$low-lying baryon resonances and $1 / 2^{+}$groundstate baryon poles, respectively. The estimation of pole amplitudes is a challenging and nontrivial task since weak baryon matrix elements and strong coupling constants of $\frac{1}{2}+$ and $\frac{1}{2}-$ baryon states are involved. As a consequence, the nonfactorizable contribution evaluated from pole diagrams is far more uncertain than the factorizable terms. The difficulty emerges in particular for $S$-wave terms as they require the information of the troublesome negative-parity baryon resonances, which are not well understood in the quark model. Fortunately, the trick of current algebra helps to avoid evaluation involving $\frac{1-}{2}$ state in the soft-meson limit [24-28]. Although the pseudoscalar meson produced in $\mathcal{B}_{c} \rightarrow \mathcal{B}+P$ decays is in general not truly soft, the current algebra approach seems to work empirically well for $\Lambda_{c}^{+} \rightarrow \mathcal{B}+P$ decays [27,28]. Moreover, the predicted negative decay asymmetries by current algebra for both $\Lambda_{c}^{+} \rightarrow \Sigma^{+} \pi^{0}$ and $\Sigma^{0} \pi^{+}$agree in sign with the recent BESIII measurements [29] (again see [27,28] for details). In contrast, the pole model or the covariant quark model and its variant always lead to a positive decay asymmetry for the aforementioned two modes. Therefore, in this work, we shall follow $[27,28,30]$ to work out the nonfactorizable $S$-wave amplitudes in $\Omega_{c}$ decays using the current algebra and the nonfactorizable contributions, including the $W$-exchange contribution, to $P$-wave ones using the pole model.

This paper is organized as follows. In Sec. II, we set up the framework for the analysis of hadronic weak decays of the singly charmed baryon $\Omega_{c}$, including the topological diagrams and the formalism for describing factorizable and nonfactorizable terms in the pole model. In Sec. III, numerical results and discussions are presented. A conclusion will be given in Sec. IV. Details on commutators in $S$-wave amplitudes are given in Appendix A. Baryon matrix elements and axial-vector form factors calculated in the MIT bag model are summarized in Appendix B and Appendix C, respectively.

\section{THEORETICAL FRAMEWORK}

In this work, we will continue studying weak decays of the $\Omega_{c}$ baryon in the topological-diagram approach, within which the factorizable and nonfactorizable contributions can be classified explicitly by topological diagrams $[20,24]$. Then, different methods are adopted to calculate the two parts of contributions separately. The factorizable amplitudes are evaluated by naive factorization, while the pole model associated with the current algebra technique is applied in the calculation of nonfactorizable amplitudes.

\section{A. Topological diagrams}

The general formulation of the topological-diagram scheme for the nonleptonic weak decays of baryons was proposed by Chau, Tseng, and Cheng more than two decades ago [31], which was then applied to all the decays of the antitriplet and sextet charmed baryons. Here, we should emphasize that the topological diagram is not identical to the Feynman diagram, an example of which can be found in [32]. In the topological-diagram approach, even when final-state rescattering is included, we can still classify the diagrams according to their topology. In charmed meson decays, the extraction of the topological diagrams from the experimental data of Cabibbo-favored (CF) channels, together with SU(3) symmetry, allows us to predict branching fractions of singly Cabibbo-suppressed (SCS) and doubly Cabibbo-suppressed (DCS) decays and even $C P$ violation. For the charmed baryon decays, however, there are not adequate data on branching fractions and decay asymmetries to extract the topological diagrams. Nevertheless, we can still use topological diagrams to identify factorizable and nonfactorizable decay amplitudes.

For the weak decays $\Omega_{c} \rightarrow \mathcal{B}+P$ ( $\mathcal{B}$ is baryon octet) of interest in this work, the relevant topological diagrams are the external $W$-emission $T$, the internal $W$-emission $C$, the inner $W$-emission $C^{\prime}$, and the $W$-exchange diagrams $E_{1}$ as well as $E_{2}$ as depicted in Fig. 1. Among them, $T$ and $C$ are factorizable, ${ }^{1}$ while $C^{\prime}$ and $W$ exchange give nonfactorizable contributions. The relevant topological diagrams for all decay modes of $\Omega_{c}$, including CF, SCS, and DCS modes, are shown in Table I. ${ }^{2}$

We notice from Table I that (i) among all the CF, SCS, and DCS decays of $\Omega_{c}$, there is no purely factorizable mode, (ii) the modes containing $\Xi^{0}$ or $\Xi^{-}$in final states receive both factorizable and nonfactorizable contributions, while the modes containing $\Sigma$ and $\Lambda^{0}$ in final states have purely nonfactorizable contribution, (iii) the $W$-exchange contribution is absent in the CF process but manifests in all the SCS and DCS decays, and (iv) the two necleons $n$ and $p$, as parts of the baryon octet, are absent in all the $\Omega_{c}$ decays.

\section{B. Kinematics}

The two-body weak decay $\mathcal{B}_{i} \rightarrow \mathcal{B}_{f} P$ generally can be parametrized by both $S$ and $P$ amplitudes, giving

\footnotetext{
${ }^{1}$ Strictly speaking, there are nonfactorizable contributions in the two diagrams. However, in charm physics these nonfactorizable contributions can be absorbed by an effective $N$ in the effective Wilson coefficients $a_{1,2}=c_{1,2}+c_{2,1} / N$ [see Eq. (9) as an example], and the value of $N$ can be extracted from the data. In that sense, the form of naive factorization can be kept, and hence, $T$ and $C$ can be classified into factorizable ones.

${ }^{2}$ The reason for neglecting the so-called type-III diagram in [18] or (d1), (d2) diagrams in [33] can be referred to [28].
} 

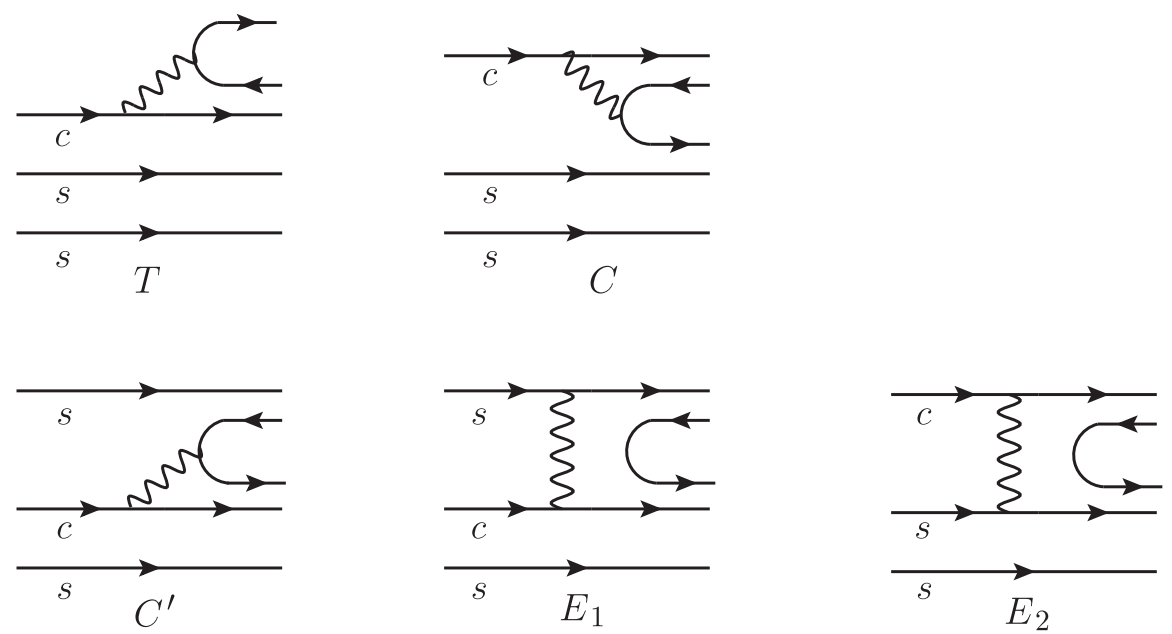

FIG. 1. Topological diagrams contributing to $\Omega_{c} \rightarrow \mathcal{B}+P$ decays: external $W$-emission $T$, internal $W$-emission $C$, inner $W$-emission $C^{\prime}, W$-exchange diagrams $E_{1}$ and $E_{2}$.

$$
M\left(\mathcal{B}_{i} \rightarrow \mathcal{B}_{f} P\right)=i \bar{u}_{f}\left(A-B \gamma_{5}\right) u_{i}
$$

Here, $\mathcal{B}_{i}$ is actually $\Omega_{c}$, the final state baryon $\mathcal{B}_{f}$ is a baryon octet, and $P$ is a pseudoscalar meson. The decay width and up-down decay asymmetry are given by

$\Gamma=\frac{p_{c}}{8 \pi}\left[\frac{\left(m_{i}+m_{f}\right)^{2}-m_{P}^{2}}{m_{i}^{2}}|A|^{2}+\frac{\left(m_{i}-m_{f}\right)^{2}-m_{P}^{2}}{m_{i}^{2}}|B|^{2}\right]$,

$\alpha=\frac{2 \kappa \operatorname{Re}\left(A^{*} B\right)}{|A|^{2}+\kappa^{2}|B|^{2}}$,

where $p_{c}$ is the three-momentum in the rest frame of the mother particle and $\kappa=p_{c} /\left(E_{f}+m_{f}\right)=$ $\sqrt{\left(E_{f}-m_{f}\right) /\left(E_{f}+m_{f}\right)}$. The $S$ - and $P$-wave amplitudes of the two-body decay generally receive both factorizable and nonfactorizable contributions,

$$
A=A^{\mathrm{fac}}+A^{\mathrm{nf}}, \quad B=B^{\mathrm{fac}}+B^{\mathrm{nf}} .
$$

We will discuss the details of factorizable and nonfactorizable amplitudes separately in below.

\section{Factorizable amplitudes}

Here, the state-of-the-art effective Hamiltonian approach is adopted to describe factorizable contributions in the charmed baryon decays. Physical observables can be expressed as products of short-distance and long-distance parameters. The short-distance physics is characterized by Wilson coefficients, which are obtained from integrating out high energy part of theory, and the long-distance physics lies in the hadronic matrix elements.

\section{General expression of factorizable amplitudes}

The effective Hamiltonian for a CF process in $\Omega_{c}$ decay is

$$
\mathcal{H}_{\text {eff }}=\frac{G_{F}}{\sqrt{2}} V_{c s} V_{u d}^{*}\left(c_{1} O_{1}+c_{2} O_{2}\right)+\text { H.c. },
$$

where the four-quark operators are given by

\begin{tabular}{|c|c|c|c|c|c|}
\hline $\mathrm{CF}$ & Contributions & SCS & Contributions & DSC & Contributions \\
\hline \multirow[t]{8}{*}{$\overline{\Omega_{c}^{0} \rightarrow \Xi^{0} \bar{K}^{0}}$} & $C, C^{\prime}$ & $\Omega_{c}^{0} \rightarrow \Xi^{-} \pi^{+}$ & $T, E_{1}$ & $\Omega_{c}^{0} \rightarrow \Xi^{0} K^{0}$ & $C, E_{2}$ \\
\hline & & $\Omega_{c}^{0} \rightarrow \Sigma^{+} K^{-}$ & $E_{2}$ & $\Omega_{c}^{0} \rightarrow \Sigma^{0} \eta$ & $C^{\prime}, E_{1}, E_{2}$ \\
\hline & & $\Omega_{c}^{0} \rightarrow \Sigma^{0} \bar{K}^{0}$ & $C^{\prime}, E_{2}$ & $\Omega_{c}^{0} \rightarrow \Lambda^{0} \eta$ & $C^{\prime}, E_{1}, E_{2}$ \\
\hline & & $\Omega_{c}^{0} \rightarrow \Lambda^{0} \bar{K}^{0}$ & $C^{\prime}, E_{2}$ & $\Omega_{c}^{0} \rightarrow \Sigma^{0} \pi^{0}$ & $E_{1}, E_{2}$ \\
\hline & & $\Omega_{c}^{0} \rightarrow \Xi^{0} \pi^{0}$ & $C, E_{1}$ & $\Omega_{c}^{0} \rightarrow \Lambda^{0} \pi^{0}$ & $E_{1}, E_{2}$ \\
\hline & & & & $\Omega_{c}^{0} \rightarrow \Xi^{-} K^{+}$ & $T, E_{1}$ \\
\hline & & & & $\Omega_{c}^{0} \rightarrow \Sigma^{+} \pi^{-}$ & $E_{2}$ \\
\hline & & & & $\Omega_{c}^{0} \rightarrow \Sigma^{-} \pi^{+}$ & $E_{1}$ \\
\hline
\end{tabular}

TABLE I. Topological diagrams contributing to two-body weak decays $\Omega_{c} \rightarrow \mathcal{B P}$, where $\mathcal{B}$ is a baryon octet and $P$ is a pseudoscalar meson. 


$$
O_{1}=(\bar{s} c)(\bar{u} d), \quad O_{2}=(\bar{u} c)(\bar{s} d)
$$

with $\left(\bar{q}_{1} q_{2}\right) \equiv \bar{q}_{1} \gamma_{\mu}\left(1-\gamma_{5}\right) q_{2}$. The Wilson coefficients to the leading order are given by $c_{1}=1.346$ and $c_{2}=-0.636$ at $\mu=1.25 \mathrm{GeV}$ and $\Lambda_{\mathrm{MS}}^{(4)}=325 \mathrm{MeV}$ [34]. Under naive factorization, the amplitude can be written down as

$$
\begin{aligned}
M & =\left\langle\bar{K}^{0} \Xi^{0}\left|\mathcal{H}_{\mathrm{eff}}\right| \Omega_{c}\right\rangle \\
& =\frac{G_{F}}{\sqrt{2}} V_{c s} V_{u d}^{*} a_{2}\left\langle\bar{K}^{0}|(\bar{s} d)| 0\right\rangle\left\langle\Xi^{0}|(\bar{u} c)| \mathcal{B}_{c}\right\rangle,
\end{aligned}
$$

where $a_{2}=c_{2}+\frac{c_{1}}{N}$. The value of $N$ is taken as $N \approx 7$, following our previous study in [27]. We also give the definition of $a_{1}$ as $a_{1}=c_{1}+\frac{c_{2}}{N}$ for the purpose of describing SCS and DCS decays conveniently. In terms of the decay constant,

$$
\left\langle K(q)\left|\bar{s}_{\mu}\left(1-\gamma_{5}\right) d\right| 0\right\rangle=i f_{K} q_{\mu},
$$

and baryon-baryon transition form factors defined by

$$
\begin{aligned}
& \left\langle\Xi^{0}\left(p_{2}\right)\left|\bar{c}_{\mu}\left(1-\gamma_{5}\right) u\right| \Omega_{c}\left(p_{1}\right)\right\rangle \\
& =\bar{u}_{2}\left[f_{1}\left(q^{2}\right) \gamma_{\mu}-f_{2}\left(q^{2}\right) i \sigma_{\mu \nu} \frac{q^{\nu}}{M}+f_{3}\left(q^{2}\right) \frac{q_{\mu}}{M}\right. \\
& \left.\quad-\left(g_{1}\left(q^{2}\right) \gamma_{\mu}-g_{2}\left(q^{2}\right) i \sigma_{\mu \nu} \frac{q^{\nu}}{M}+g_{3}\left(q^{2}\right) \frac{q_{\mu}}{M}\right) \gamma_{5}\right] u_{1},
\end{aligned}
$$

with the momentum transfer $q=p_{1}-p_{2}$, we obtain the factorizable amplitude as

$$
\begin{aligned}
M\left(\Omega_{c}\right. & \left.\rightarrow \Xi^{0} \bar{K}^{0}\right) \\
= & i \frac{G_{F}}{\sqrt{2}} a_{2} V_{u d}^{*} V_{c s} f_{K} \bar{u}_{2}\left(p_{2}\right)\left[\left(m_{1}-m_{2}\right) f_{1}\left(q^{2}\right)\right. \\
& \left.+\left(m_{1}+m_{2}\right) g_{1}\left(q^{2}\right) \gamma_{5}\right] u_{1}\left(p_{1}\right) .
\end{aligned}
$$

Contributions from the form factors $f_{3}$ and $g_{3}$ have been neglected with the similar reason given in the footnote 1 of [30]. More explicitly, the factorizable $S$ - and $P$-wave amplitudes read

$$
\begin{aligned}
\left.A^{\mathrm{fac}}\right|_{\mathrm{CF}} & =\frac{G_{F}}{\sqrt{2}} a_{2} V_{u d}^{*} V_{c s} f_{K}\left(m_{\Omega_{c}}-m_{\Xi^{0}}\right) f_{1}\left(q^{2}\right), \\
\left.B^{\mathrm{fac}}\right|_{\mathrm{CF}} & =-\frac{G_{F}}{\sqrt{2}} a_{2} V_{u d}^{*} V_{c s} f_{K}\left(m_{\Omega_{c}}+m_{\Xi^{0}}\right) g_{1}\left(q^{2}\right) .
\end{aligned}
$$

Likewise, the $S$ - and $P$-wave amplitudes for SCS processes and DCS processes are given, respectively, by

$\begin{aligned}\left.A^{\mathrm{fac}}\right|_{\mathrm{SCS}} & =\frac{G_{F}}{\sqrt{2}} a_{1,2} V_{u q}^{*} V_{c q} f_{P}\left(m_{\Omega_{c}}-m_{\mathcal{B}}\right) f_{1}\left(q^{2}\right), \\ \left.B^{\mathrm{fac}}\right|_{\mathrm{SCS}} & =-\frac{G_{F}}{\sqrt{2}} a_{1,2} V_{u q}^{*} V_{c q} f_{P}\left(m_{\Omega_{c}}+m_{\mathcal{B}}\right) g_{1}\left(q^{2}\right),\end{aligned}$

and

$\begin{aligned}\left.A^{\mathrm{fac}}\right|_{\mathrm{DCS}} & =\frac{G_{F}}{\sqrt{2}} a_{1,2} V_{u s}^{*} V_{c d} f_{P}\left(m_{\Omega_{c}}-m_{\mathcal{B}}\right) f_{1}\left(q^{2}\right), \\ \left.B^{\mathrm{fac}}\right|_{\mathrm{DCS}} & =-\frac{G_{F}}{\sqrt{2}} a_{1,2} V_{u s}^{*} V_{c d} f_{P}\left(m_{\Omega_{c}}+m_{\mathcal{B}}\right) g_{1}\left(q^{2}\right),\end{aligned}$

where the choice of $a_{i}$ depends on the meson in final states. In the amplitude of SCS processes, Eq. (14) the flavor of the down-type quark $q(q=d$ or $s)$ depends on an individual process.

\section{Baryon transition form factors}

The baryon transition form factor (FF) parameter characterizes the transition between two baryons and is calculated nonperturbatively. There have been some works on $\Omega_{c}-\mathcal{B}$ transition FFs, including the nonrelativistic quark model (NRQM) [35], heavy quark effective theory (HQET) [36], as well as the light-front quark model (LFQM) [23]. As we have emphasized that the nonfactorizable contribution gives significant contribution to the decay rates and decay asymmetries, the relative sign between factorizable and nonfactorizable amplitudes is critical. Hence, an estimation of FFs as well as baryon matrix elements in a self-consistent convention is desirable. In this work, we shall work out both FFs and baryonic matrix elements in the framework of the MIT bag model [37].

Following [24], we can write down the $q^{2}$ dependence of $\mathrm{FF}$ as

$$
f_{i}\left(q^{2}\right)=\frac{f_{i}(0)}{\left(1-q^{2} / m_{V}^{2}\right)^{2}}, \quad g_{i}\left(q^{2}\right)=\frac{g_{i}(0)}{\left(1-q^{2} / m_{A}^{2}\right)^{2}},
$$

where $m_{V}=2.01 \mathrm{GeV}, m_{A}=2.42 \mathrm{GeV}$ for the $(c \bar{d})$ quark content, and $m_{V}=2.11 \mathrm{GeV}, m_{A}=2.54 \mathrm{GeV}$ for $(c \bar{s})$ quark content. In the zero recoil limit, where $q_{\max }^{2}=\left(m_{i}-m_{f}\right)^{2}$, FFs can be calculated in the MIT bag model [20], giving

$$
\begin{aligned}
& f_{1}^{\mathcal{B}_{f} \mathcal{B}_{i}}\left(q_{\text {max }}^{2}\right)=\left\langle\mathcal{B}_{f}^{\uparrow}\left|b_{q_{1}}^{\dagger} b_{q_{2}}\right| \mathcal{B}_{i}^{\uparrow}\right\rangle \int d^{3} \boldsymbol{r}\left(u_{q_{1}}(r) u_{q_{2}}(r)+v_{q_{1}}(r) v_{q_{2}}(r)\right), \\
& g_{1}^{\mathcal{B}_{f} \mathcal{B}_{i}}\left(q_{\text {max }}^{2}\right)=\left\langle\mathcal{B}_{f}^{\uparrow}\left|b_{q_{1}}^{\dagger} b_{q_{2}} \sigma_{z}\right| \mathcal{B}_{i}^{\uparrow}\right\rangle \int d^{3} \boldsymbol{r}\left(u_{q_{1}}(r) u_{q_{2}}(r)-\frac{1}{3} v_{q_{1}}(r) v_{q_{2}}(r)\right),
\end{aligned}
$$


TABLE II. The calculated form factors in the MIT bag model at maximum four-momentum transfer squared $q^{2}=q_{\text {max }}^{2}=\left(m_{i}-m_{f}\right)^{2}$ and $q^{2}=m_{P}^{2}$.

\begin{tabular}{lccccccc}
\hline \hline Modes & $(c \bar{q})$ & $f_{1}\left(q_{\max }^{2}\right)$ & $f_{1}\left(m_{P}^{2}\right) / f_{1}\left(q_{\max }^{2}\right)$ & $f_{1}\left(m_{P}^{2}\right)$ & $g_{1}\left(q_{\max }^{2}\right)$ & $g_{1}\left(m_{P}^{2}\right) / g_{1}\left(q_{\max }^{2}\right)$ & $g_{1}\left(m_{P}^{2}\right)$ \\
\hline$\Omega_{c}^{0} \rightarrow \Xi^{0} \bar{K}^{0}$ & $(c \bar{s})$ & $Y_{1}$ & 0.37 & 0.32 & $-\frac{1}{3} Y_{2}$ & 0.54 & -0.14 \\
$\Omega_{c}^{0} \rightarrow \Xi^{-} \pi^{+}$ & $(c \bar{d})$ & $Y_{1}$ & 0.29 & 0.25 & $-\frac{1}{3} Y_{2}$ & 0.46 & -0.12 \\
$\Omega_{c}^{0} \rightarrow \Xi^{0} \pi^{0}$ & $(c \bar{d})$ & $Y_{1}$ & 0.28 & 0.25 & $-\frac{1}{3} Y_{2}$ & 0.46 & -0.12 \\
$\Omega_{c}^{0} \rightarrow \Xi^{0} K^{0}$ & $(c \bar{d})$ & $Y_{1}$ & 0.32 & 0.28 & $-\frac{1}{3} Y_{2}$ & 0.50 & -0.13 \\
$\Omega_{c}^{0} \rightarrow \Xi^{-} K^{+}$ & $(c \bar{d})$ & $Y_{1}$ & 0.32 & 0.28 & $-\frac{1}{3} Y_{2}$ & 0.50 & -0.13 \\
\hline \hline
\end{tabular}

where $u(r)$ and $v(r)$ are the large and small components, respectively, of the quark wave function in the bag model. FFs at different $q^{2}$ are related via

$$
\begin{aligned}
& f_{i}\left(q_{2}^{2}\right)=\frac{\left(1-q_{1}^{2} / m_{V}^{2}\right)^{2}}{\left(1-q_{2}^{2} / m_{V}^{2}\right)^{2}} f_{i}\left(q_{1}^{2}\right), \\
& g_{i}\left(q_{2}^{2}\right)=\frac{\left(1-q_{1}^{2} / m_{A}^{2}\right)^{2}}{\left(1-q_{2}^{2} / m_{A}^{2}\right)^{2}} g_{i}\left(q_{1}^{2}\right) .
\end{aligned}
$$

This allows us to obtain the physical FFs at $q^{2}=m_{P}^{2}$.

It is obvious that the FF at $q_{\max }^{2}$ is determined only by the baryons in initial and final states. However, its evolution with $q^{2}$ is governed by both the final-state meson and relevant quark content. Such a dependence is reflected in Table II, in which the quark contents are shown in the second column. In the zero recoil limit, the FFs at $q_{\max }^{2}$ calculated from Eq. (16) are presented in the third and sixth columns. And then in the fourth and seventh columns, the evolution of FFs from $q^{2}=q_{\max }^{2}$ to $q^{2}=m_{P}^{2}$ are derived according to Eq. (18). The auxiliary quantities $Y_{1,2}$ can be obtained from the calculation in MIT bag model, giving

$$
\begin{aligned}
& Y_{1}=4 \pi \int r^{2} d r\left(u_{u} u_{c}+v_{u} v_{c}\right), \\
& Y_{1}^{s}=4 \pi \int r^{2} d r\left(u_{s} u_{c}+v_{s} v_{c}\right), \\
& Y_{2}=4 \pi \int r^{2} d r\left(u_{u} u_{c}-\frac{1}{3} v_{u} v_{c}\right), \\
& Y_{2}^{s}=4 \pi \int r^{2} d r\left(u_{s} u_{c}-\frac{1}{3} v_{s} v_{c}\right) .
\end{aligned}
$$

The model parameters are adopted from [27] and references therein. Numerically, we have $Y_{1}=0.88, \quad Y_{1}^{s}=0.95$,
$Y_{2}=0.77$, and $Y_{2}^{s}=0.86$, which are consistent with the corresponding numbers in [20].

A comparison is made in Table III among results of $\Omega_{c} \rightarrow \Xi \mathrm{FF}$ in various approaches at $q^{2}=0$. From Eq. (16) and Table II, FFs at $q^{2}=q_{\max }^{2}$ are identical for $\Omega_{c} \rightarrow \Xi^{0,-} P$. However, the values would be changed at a different energy scale and also depend on different meson states. In this comparison, we ignore such slight differences. Note that all the nonperturbative quantities involving in this work are calculated in the MIT bag model; thus, our convention of signs should be consistent globally. For this reason, we correct the signs in both NRQM and HQET cases. Apparently, NRQM gives small values for both $f_{1}$ and $g_{1}$, while the predictions from LFQM are the largest.

\section{Nonfactorizable amplitudes}

Now it is widely accepted that in charmed baryon decays nonfactorizable amplitude can not be neglected, sometimes even gives dominated contributions to branching fraction and decay asymmetry in particular processes. In the topological-diagram approach, these nonfactorizable contributions have been presented by topological diagrams $C^{\prime}, E_{1}$, and $E_{2}$ in Fig. 1. A further calculation of nonfactorizable contribution relies on the pole model, while the pole diagrams will be obtained by the correspondence between topological diagrams [20].

The general formula for $S$ - and $P$-wave nonfactorizable amplitudes in the pole model is given by $[27,28,30]$

\begin{tabular}{|c|c|c|c|c|c|c|c|c|}
\hline \multirow[b]{2}{*}{$\Omega_{c} \rightarrow \mathcal{B}$} & \multicolumn{4}{|c|}{$f_{1}(0)$} & \multicolumn{4}{|c|}{$g_{1}(0)$} \\
\hline & MIT & NRQM & HQET & LFQM & MIT & NRQM & HQET & LFQM \\
\hline$\overline{\Omega_{c}^{0} \rightarrow \Xi}$ & 0.34 & 0.23 & 0.34 & 0.653 & -0.15 & -0.14 & -0.10 & -0.182 \\
\hline
\end{tabular}

$$
\begin{aligned}
A^{\text {pole }} & =-\sum_{B_{n}^{*}\left(1 / 2^{-}\right)}\left[\frac{g_{B_{f} B_{n}^{*} M} b_{n^{*} i}}{m_{i}-m_{n^{*}}}+\frac{b_{f n^{*}} g_{B_{n}^{*} B_{i} M}}{m_{f}-m_{n^{*}}}\right], \\
B^{\text {pole }} & =\sum_{B_{n}}\left[\frac{g_{B_{f} B_{n} M} a_{n i}}{m_{i}-m_{n}}+\frac{a_{f n} g_{B_{n} B_{i} M}}{m_{f}-m_{n}}\right],
\end{aligned}
$$

TABLE III. Form factors $f_{1}\left(q^{2}\right)$ and $g_{1}\left(q^{2}\right)$ at $q^{2}=0$ for $\Omega_{c}^{0} \rightarrow \Xi$ transitions evaluated in the MIT bag model (this work), nonrelativistic quark model (NRQM) [35], and heavy quark effective theory (HQET) [36], which were quoted in [22], as well as the light-front quark model (LFQM) [23]. 
with the baryonic matrix elements,

$$
\begin{aligned}
\left\langle\mathcal{B}_{n}|H| \mathcal{B}_{i}\right\rangle & =\bar{u}_{n}\left(a_{n i}+b_{n i} \gamma_{5}\right) u_{i}, \\
\left\langle\mathcal{B}_{i}^{*}\left(1 / 2^{-}\right)|H| \mathcal{B}_{j}\right\rangle & =\bar{u}_{i *} b_{i^{*} j} u_{j} .
\end{aligned}
$$

To estimate the $S$-wave amplitudes in the pole model is a difficult and nontrivial task as it involves the matrix elements and strong coupling constants of $1 / 2^{-}$baryon resonances, which is less known [24]. Nevertheless, provided a soft emitted pseudoscalar meson, the intermediate excited baryons can be summed up, leading to a commutator term,

$A^{\mathrm{com}}=-\frac{\sqrt{2}}{f_{P^{a}}}\left\langle\mathcal{B}_{f}\left|\left[Q_{5}^{a}, H_{\mathrm{eff}}^{\mathrm{PV}}\right]\right| \mathcal{B}_{i}\right\rangle=\frac{\sqrt{2}}{f_{P^{a}}}\left\langle\mathcal{B}_{f}\left|\left[Q^{a}, H_{\mathrm{eff}}^{\mathrm{PC}}\right]\right| \mathcal{B}_{i}\right\rangle$,

with

$Q^{a}=\int d^{3} x \bar{q} \gamma^{0} \frac{\lambda^{a}}{2} q, \quad Q_{5}^{a}=\int d^{3} x \bar{q} \gamma^{0} \gamma_{5} \frac{\lambda^{a}}{2} q$

Likewise, the $P$-wave amplitude is reduced in the softmeson limit to

$B^{\mathrm{ca}}=\frac{\sqrt{2}}{f_{P^{a}}} \sum_{\mathcal{B}_{n}}\left[g_{\mathcal{B}_{f} \mathcal{B}_{n}}^{A} \frac{m_{f}+m_{n}}{m_{i}-m_{n}} a_{n i}+a_{f n} \frac{m_{i}+m_{n}}{m_{f}-m_{n}} g_{\mathcal{B}_{n} \mathcal{B}_{i}}^{A}\right]$, where we have applied the generalized GoldbergerTreiman relation,

$$
g_{\mathcal{B}^{\prime} \mathcal{B} P^{a}}=\frac{\sqrt{2}}{f_{P^{a}}}\left(m_{\mathcal{B}}+m_{\mathcal{B}^{\prime}}\right) g_{\mathcal{B}^{\prime} \mathcal{B}}^{A}
$$

Equations (22) and (24) are the master equations for nonfactorizable amplitudes in the pole model under the soft meson approximation.

\section{S-wave amplitudes}

We have demonstrated that $S$-wave amplitudes can be simplified to baryon matrix elements of a set of commutators in the limit of a soft meson; see Eq. (22). In Appendix A, more explicit expressions for commutators corresponding to different final states are given. The remaining task is then to evaluate different sets of commutators.

We shall present our results after a straightforward calculation, for the $\mathrm{S}$-wave amplitudes, as follows:

$$
A^{\mathrm{com}}\left(\Omega_{c}^{0} \rightarrow \Xi^{0} \bar{K}^{0}\right)=-\frac{\sqrt{2}}{f_{K}} a_{\Xi^{0} \Xi_{c}^{\prime \prime}}
$$

for the Cabibbo-favored (CF) process,

$$
\begin{aligned}
& A^{\mathrm{com}}\left(\Omega_{c}^{0} \rightarrow \Xi^{-} \pi^{+}\right)=\frac{1}{f_{\pi}} a_{\Xi^{0} \Omega_{c}^{0}}, \quad A^{\mathrm{com}}\left(\Omega_{c}^{0} \rightarrow \Sigma^{+} K^{-}\right)=\frac{1}{f_{K}}\left(a_{\Xi^{0} \Omega_{c}^{0}}-\sqrt{2} a_{\Sigma^{+} \Xi_{c}^{\prime+}}\right), \\
& A^{\mathrm{com}}\left(\Omega_{c}^{0} \rightarrow \Sigma^{0} \bar{K}^{0}\right)=\frac{1}{f_{K}}\left(-\frac{\sqrt{2}}{2} a_{\Xi^{0} \Omega_{c}^{0}}-\sqrt{2} a_{\Sigma^{0} \Xi_{c}^{\prime 0}}\right), \quad A^{\mathrm{com}}\left(\Omega_{c}^{0} \rightarrow \Xi^{0} \pi^{0}\right)=\frac{1}{f_{\pi}} \frac{\sqrt{2}}{2} a_{\Xi^{0} \Omega_{c}^{0}}, \\
& A^{\mathrm{com}}\left(\Omega_{c}^{0} \rightarrow \Lambda^{0} \bar{K}^{0}\right)=\frac{1}{f_{K}}\left(\frac{\sqrt{6}}{2} a_{\Xi^{0} \Omega_{c}^{0}}-\sqrt{2} a_{\Lambda^{0} \Xi_{c}^{0}}\right),
\end{aligned}
$$

for singly Cabibbo-suppressed (SCS) processes, and

$$
\begin{aligned}
A^{\operatorname{com}}\left(\Omega_{c}^{0} \rightarrow \Xi^{0} K^{0}\right) & =\frac{1}{f_{K}}\left(-\frac{\sqrt{2}}{2} a_{\Sigma^{0} \Omega_{c}^{0}}+\frac{\sqrt{6}}{2} a_{\Lambda \Omega_{c}^{0}}\right), \quad A^{\operatorname{com}}\left(\Omega_{c}^{0} \rightarrow \Sigma^{0} \eta\right)=\frac{\sqrt{6}}{f_{\eta_{8}}} a_{\Sigma^{0} \Omega_{c}^{0}}, \\
A^{\operatorname{com}}\left(\Omega_{c}^{0} \rightarrow \Lambda^{0} \eta\right) & =\frac{\sqrt{6}}{f_{\eta_{8}}} a_{\Lambda^{0} \Omega_{c}^{0}}, \quad A^{\operatorname{com}}\left(\Omega_{c}^{0} \rightarrow \Sigma^{-} \pi^{+}\right)=\frac{\sqrt{2}}{f_{\pi}} a_{\Sigma^{0} \Omega_{c}^{0}}, \\
A^{\operatorname{com}}\left(\Omega_{c}^{0} \rightarrow \Xi^{-} K^{+}\right) & =-\frac{1}{f_{K}}\left(\frac{\sqrt{2}}{2} a_{\Sigma^{0} \Omega_{c}^{0}}+\frac{\sqrt{6}}{2} a_{\Lambda \Omega_{c}^{0}}\right), \quad A^{\operatorname{com}}\left(\Omega_{c}^{0} \rightarrow \Sigma^{+} \pi^{-}\right)=-\frac{\sqrt{2}}{f_{\pi}} a_{\Sigma^{0} \Omega_{c}^{0}}, \\
A^{\operatorname{com}}\left(\Omega_{c}^{0} \rightarrow \Sigma^{0} \pi^{0}\right) & =0, \quad A^{\operatorname{com}}\left(\Omega_{c}^{0} \rightarrow \Lambda^{0} \pi^{0}\right)=0,
\end{aligned}
$$

for the doubly Cabibbo-suppressed (DCS) processes, where the baryonic matrix element $\left\langle\mathcal{B}^{\prime}\left|H_{\mathrm{eff}}^{\mathrm{PC}}\right| \mathcal{B}\right\rangle$ is denoted by $a_{\mathcal{B}^{\prime} \mathcal{B}}$. We find that a straightforward calculation of the last two terms directly leads to vanishing results. This can be easily understood as $I_{3}\left(\Lambda^{0}\right)=I_{3}\left(\Sigma^{0}\right)=0$. 


\section{P-wave amplitudes}

Now we turn to the nonfactorizable $P$-wave amplitudes given by Eq. (24). By substituting explicit hadron states, we have

$$
B^{\mathrm{ca}}\left(\Omega_{c}^{0} \rightarrow \Xi^{0} \bar{K}^{0}\right)=\frac{1}{f_{K}}\left(a_{\Xi^{0} \Xi_{c}^{0}} \frac{m_{\Omega_{c}^{0}}+m_{\Xi_{c}^{0}}}{m_{\Xi^{0}}-m_{\Xi_{c}^{0}}} g_{\Xi_{c}^{0} \Omega_{c}^{0}}^{A\left(\bar{K}^{0}\right)}+a_{\Xi^{0} \Xi_{c}^{00}} \frac{m_{\Omega_{c}^{0}}+m_{\Xi_{c}^{\prime 0}}}{m_{\Xi^{0}}-m_{\Xi_{c}^{\prime 0}}} g_{\Xi_{c}^{\prime \prime} \Omega_{c}^{0}}^{A\left(\bar{R}^{0}\right)}\right),
$$

for CF decays,

$$
\begin{aligned}
B^{\mathrm{ca}}\left(\Omega_{c}^{0} \rightarrow \Xi^{-} \pi^{+}\right) & =\frac{1}{f_{\pi}}\left(g_{\Xi^{-} \Xi^{0}}^{A\left(\pi^{+}\right)} \frac{m_{\Xi^{-}}+m_{\Xi^{0}}}{m_{\Omega_{c}^{0}}-m_{\Xi^{0}}} a_{\Xi^{0} \Omega_{c}^{0}}\right), \\
B^{\mathrm{ca}}\left(\Omega_{c}^{0} \rightarrow \Sigma^{+} K^{-}\right) & =\frac{1}{f_{K}}\left(g_{\Sigma^{+} \Xi^{0}}^{A\left(K^{-}\right)} \frac{m_{\Sigma^{+}}+m_{\Xi^{0}}}{m_{\Omega_{c}^{0}}-m_{\Xi^{0}}} a_{\Xi^{0} \Omega_{c}^{0}}\right), \\
B^{\mathrm{ca}}\left(\Omega_{c}^{0} \rightarrow \Sigma^{0} \bar{K}^{0}\right) & =\frac{1}{f_{K}}\left(a_{\Sigma^{0} \Xi_{c}^{0}} \frac{m_{\Omega_{c}^{0}}+m_{\Xi_{c}^{0}}}{m_{\Sigma^{0}}-m_{\Xi_{c}^{0}}} g_{\Xi_{c}^{0} \Omega_{c}^{0}}^{A\left(\bar{K}^{0}\right)}+a_{\Sigma^{0} \Xi_{c}^{\prime 0}} \frac{m_{\Omega_{c}^{0}}+m_{\Xi_{c}^{\prime 0}}}{m_{\Sigma^{0}}-m_{\Xi_{c}^{\prime 0}}} g_{\Xi_{c}^{\prime} \Omega_{c}^{0}}^{A\left(\bar{K}^{0}\right)}+g_{\Sigma^{0} \Xi^{0}}^{A\left(\bar{K}^{0}\right)} \frac{m_{\Sigma^{0}}+m_{\Xi^{0}}}{m_{\Omega_{c}^{0}}-m_{\Xi^{0}}} a_{\Xi^{0} \Omega_{c}^{0}}\right), \\
B^{\mathrm{ca}}\left(\Omega_{c}^{0} \rightarrow \Lambda^{0} \bar{K}^{0}\right) & =\frac{1}{f_{K}}\left(a_{\Lambda^{0} \Xi_{c}^{0}} \frac{m_{\Omega_{c}^{0}}+m_{\Xi_{c}^{0}}^{0}}{m_{\Lambda^{0}}-m_{\Xi_{c}^{0}}} g_{\Xi_{c}^{0} \Omega_{c}^{0}}^{A\left(\bar{K}^{0}\right)}+a_{\Lambda^{0} \Xi_{c}^{\prime \prime}} \frac{m_{\Omega_{c}^{0}}+m_{\Xi_{c}^{\prime 0}}}{m_{\Lambda^{0}}-m_{\Xi_{c}^{\prime}}} g_{\Xi_{c}^{\prime 0} \Omega_{c}^{0}}^{A\left(\bar{K}^{0}\right)}+g_{\Lambda^{0} \Xi^{0}}^{A\left(\bar{K}^{0}\right)} \frac{m_{\Lambda^{0}}+m_{\Xi^{0}}}{m_{\Omega_{c}^{0}}-m_{\Xi^{0}}} a_{\Xi^{0} \Omega_{c}^{0}}\right), \\
B^{\mathrm{ca}}\left(\Omega_{c}^{0} \rightarrow \Xi^{0} \pi^{0}\right) & =\frac{\sqrt{2}}{f_{\pi}}\left(g_{\Xi^{0} \Xi^{0}}^{A\left(\pi^{0}\right)} \frac{m_{\Xi^{0}}+m_{\Xi^{0}}}{m_{\Omega_{c}^{0}}-m_{\Xi^{0}}} a_{\Xi^{0} \Omega_{c}^{0}}\right),
\end{aligned}
$$

for SCS processes, and

$$
\begin{aligned}
& B^{\mathrm{ca}}\left(\Omega_{c}^{0} \rightarrow \Xi^{0} K^{0}\right)=\frac{1}{f_{K}}\left(g_{\Xi^{0} \Lambda^{0}}^{A\left(K^{0}\right)} \frac{m_{\Xi^{0}}+m_{\Lambda^{0}}}{m_{\Omega_{c}^{0}}-m_{\Lambda^{0}}} a_{\Lambda^{0} \Omega_{c}^{0}}+g_{\Xi^{0} \Sigma^{0}}^{A\left(K^{0}\right)} \frac{m_{\Xi^{0}}+m_{\Sigma^{0}}}{m_{\Omega_{c}^{0}}-m_{\Sigma^{0}}} a_{\Sigma^{0} \Omega_{c}^{0}}\right), \\
& B^{\mathrm{ca}}\left(\Omega_{c}^{0} \rightarrow \Sigma^{0} \eta_{8}\right)=\frac{\sqrt{2}}{f_{\eta_{8}}}\left(a_{\Sigma^{0} \Omega_{c}^{0}} \frac{m_{\Omega_{c}^{0}}+m_{\Omega_{c}^{0}}}{m_{\Sigma^{0}}-m_{\Omega_{c}^{0}}} g_{\Omega_{c}^{0} \Omega_{c}^{0}}^{A\left(\eta_{8}\right)}+g_{\Sigma^{0} \Sigma^{0}}^{A\left(\eta_{8}\right)} \frac{m_{\Sigma^{0}}+m_{\Sigma^{0}}}{m_{\Omega_{c}^{0}}-m_{\Sigma^{0}}} a_{\Sigma^{0} \Omega_{c}^{0}}+g_{\Sigma^{0} \Lambda^{0}}^{A\left(\eta_{8}\right)} \frac{m_{\Sigma^{0}}+m_{\Lambda^{0}}}{m_{\Omega_{c}^{0}}-m_{\Lambda^{0}}} a_{\Lambda^{0} \Omega_{c}^{0}}\right), \\
& B^{\mathrm{ca}}\left(\Omega_{c}^{0} \rightarrow \Lambda^{0} \eta_{8}\right)=\frac{\sqrt{2}}{f_{\eta_{8}}}\left(a_{\Lambda^{0} \Omega_{c}^{0}} \frac{m_{\Omega_{c}^{0}}+m_{\Omega_{c}^{0}}}{m_{\Lambda^{0}}-m_{\Omega_{c}^{0}}} g_{\Omega_{c}^{0} \Omega_{c}^{0}}^{A\left(\eta_{8}\right)}+g_{\Lambda^{0} \Sigma^{0}}^{A\left(\eta_{8}\right)} \frac{m_{\Lambda^{0}}+m_{\Sigma^{0}}}{m_{\Omega_{c}^{0}}-m_{\Sigma^{0}}} a_{\Sigma^{0} \Omega_{c}^{0}}+g_{\Lambda^{0} \Lambda^{0}}^{A\left(\eta_{8}\right)} \frac{m_{\Lambda^{0}}+m_{\Lambda^{0}}}{m_{\Omega_{c}^{0}}-m_{\Lambda^{0}}} a_{\Lambda^{0} \Omega_{c}^{0}}\right), \\
& B^{\mathrm{ca}}\left(\Omega_{c}^{0} \rightarrow \Xi^{-} K^{+}\right)=\frac{1}{f_{K}}\left(g_{\Xi^{-} \Sigma^{0}}^{A\left(K^{+}\right)} \frac{m_{\Xi^{-}}+m_{\Sigma^{0}}}{m_{\Omega_{c}^{0}}-m_{\Sigma^{0}}} a_{\Sigma^{0} \Omega_{c}^{0}}+g_{\Xi^{-} \Lambda^{0}}^{A\left(K^{+}\right)} \frac{m_{\Xi^{-}}+m_{\Lambda^{0}}}{m_{\Omega_{c}^{0}}-m_{\Lambda^{0}}} a_{\Lambda^{0} \Omega_{c}^{0}}\right), \\
& B^{\mathrm{ca}}\left(\Omega_{c}^{0} \rightarrow \Sigma^{-} \pi^{+}\right)=\frac{1}{f_{\pi}}\left(g_{\Sigma^{-} \Sigma^{0}}^{A\left(\pi^{+}\right)} \frac{m_{\Sigma^{-}}+m_{\Sigma^{0}}}{m_{\Omega_{c}^{0}}-m_{\Sigma^{0}}} a_{\Sigma^{0} \Omega_{c}^{0}}+g_{\Sigma^{-} \Lambda^{0}}^{A\left(\pi^{+}\right)} \frac{m_{\Sigma^{-}}+m_{\Lambda^{0}}}{m_{\Omega_{c}^{0}}-m_{\Lambda^{0}}} a_{\Lambda^{0} \Omega_{c}^{0}}\right), \\
& B^{\mathrm{ca}}\left(\Omega_{c}^{0} \rightarrow \Sigma^{+} \pi^{-}\right)=\frac{1}{f_{\pi}}\left(g_{\Sigma^{+} \Sigma^{0}}^{A\left(\pi^{-}\right)} \frac{m_{\Sigma^{+}}+m_{\Sigma^{0}}}{m_{\Omega_{c}^{0}}-m_{\Sigma^{0}}} a_{\Sigma^{0} \Omega_{c}^{0}}+g_{\Sigma^{+} \Lambda^{0}}^{A\left(\pi^{-}\right)} \frac{m_{\Sigma^{+}}+m_{\Lambda^{0}}}{m_{\Omega_{c}^{0}}-m_{\Lambda^{0}}} a_{\Lambda^{0} \Omega_{c}^{0}}\right), \\
& B^{\mathrm{ca}}\left(\Omega_{c}^{0} \rightarrow \Sigma^{0} \pi^{0}\right)=\frac{\sqrt{2}}{f_{\pi}}\left(g_{\Sigma^{0} \Lambda^{0}}^{A\left(\pi^{0}\right)} \frac{m_{\Sigma^{0}}+m_{\Lambda^{0}}}{m_{\Omega_{c}^{0}}-m_{\Lambda^{0}}} a_{\Lambda^{0} \Omega_{c}^{0}}+g_{\Sigma^{0} \Sigma^{0}}^{A\left(\pi^{0}\right)} \frac{m_{\Sigma^{0}}+m_{\Sigma^{0}}}{m_{\Omega_{c}^{0}}-m_{\Sigma^{0}}} a_{\Sigma^{0} \Omega_{c}^{0}}\right), \\
& B^{\mathrm{ca}}\left(\Omega_{c}^{0} \rightarrow \Lambda^{0} \pi^{0}\right)=\frac{\sqrt{2}}{f_{\pi}}\left(g_{\Lambda^{0} \Lambda^{0}}^{A\left(\pi^{0}\right)} \frac{m_{\Lambda^{0}}+m_{\Lambda^{0}}}{m_{\Omega_{c}^{0}}-m_{\Lambda^{0}}} a_{\Lambda^{0} \Omega_{c}^{0}}+g_{\Lambda^{0} \Sigma^{0}}^{A\left(\pi^{0}\right)} \frac{m_{\Lambda^{0}}+m_{\Sigma^{0}}}{m_{\Omega_{c}^{0}}-m_{\Sigma^{0}}} a_{\Sigma^{0} \Omega_{c}^{0}}\right),
\end{aligned}
$$

for DCS decay processes. A furthermore derivation of nonperturbative quantities $a_{\mathcal{B B}^{\prime}}$ and $g_{\mathcal{B B}^{\prime}}^{A}$ can be found in Appendixes B and C.

\section{RESULTS AND DISCUSSION}

\section{A. Numerical results and discussions}

In this section, we shall numerically calculate branching fractions and up-down decay asymmetries. The decay asymmetries rely on $S$ - and $P$-wave amplitudes, which have been calculated analytically yet. One more parameter, lifetime, enters the calculation of branching fractions based on the decay width in Eq. (5). The value of the lifetime quoted in this work is reported by LHCb in 2018 [see Eq. (2)]. 
TABLE IV. Decays $\Omega_{c} \rightarrow \mathcal{B P}$ : the amplitudes are in units of $10^{-2} G_{F} \mathrm{GeV}^{2}$, branching fractions for CF(SCS, DCS) process(es) is (are) in unit(s) of $10^{-2}\left(10^{-3}, 10^{-4}\right)$, and the asymmetry parameters $\alpha$ are shown in the last column. ${ }^{4}$

\begin{tabular}{lccccccccc}
\hline \hline Channel & $A^{\text {fac }}$ & $A^{\text {com }}$ & $A^{\text {tot }}$ & $B^{\text {fac }}$ & $B^{\text {ca }}$ & $B^{\text {tot }}$ & $\mathcal{B}_{\text {theo }}$ & $\mathcal{B}_{\text {expt }}$ & $\alpha_{\text {theo }}$ \\
\hline$\Omega_{c}^{0} \rightarrow \Xi^{0} \bar{K}^{0}$ & -2.15 & 10.92 & 8.78 & -2.64 & 10.12 & 7.48 & 3.78 & $\cdots$ & 0.51 \\
$\Omega_{c}^{0} \rightarrow \Sigma^{+} K^{-}$ & 0 & -0.01 & -0.01 & 0 & -6.10 & -6.10 & 2.32 & $\ldots$ & 0.01 \\
$\Omega_{c}^{0} \rightarrow \Sigma^{0} \bar{K}^{0}$ & 0 & 0.01 & 0.01 & 0 & -1.21 & -1.21 & 0.09 & $\ldots$ & -0.03 \\
$\Omega_{c}^{0} \rightarrow \Lambda^{0} \bar{K}^{0}$ & 0 & -4.21 & -4.21 & 0 & 0.04 & 0.04 & 8.05 & $\ldots$ & -0.01 \\
$\Omega_{c}^{0} \rightarrow \Xi^{0} \pi^{0}$ & -0.88 & -2.43 & -3.31 & -1.21 & 1.00 & -0.21 & 5.46 & $\ldots$ & 0.04 \\
$\Omega_{c}^{0} \rightarrow \Xi^{-} \pi^{+}$ & -0.89 & -3.44 & -4.33 & -1.22 & 1.42 & 0.20 & 9.34 & $\ldots$ & -0.03 \\
$\Omega_{c}^{0} \rightarrow \Xi^{-} K^{+}$ & 0.10 & 1.34 & 1.43 & 0.13 & 0.49 & 0.62 & 9.58 & $\ldots$ & 0.27 \\
$\Omega_{c}^{0} \rightarrow \Xi^{0} K^{0}$ & 0.10 & -1.34 & -1.24 & 0.131 & -0.49 & -0.36 & 7.04 & $\ldots$ & 0.18 \\
$\Omega_{c}^{0} \rightarrow \Lambda \eta$ & 0 & -2.66 & -2.66 & 0 & -2.56 & -2.56 & 36.28 & $\ldots$ & 0.66 \\
$\Omega_{c}^{0} \rightarrow \Sigma^{0} \pi^{0}$ & 0 & 0 & 0 & 0 & -1.03 & -1.03 & 0.77 & $\ldots$ & 0 \\
$\Omega_{c}^{0} \rightarrow \Sigma^{+} \pi^{-}$ & 0 & 0 & 0 & 0 & -1.03 & -1.03 & 0.77 & $\ldots$ & 0 \\
$\Omega_{c}^{0} \rightarrow \Sigma^{-} \pi^{+}$ & 0 & 0 & 0 & 0 & -1.03 & -1.03 & 0.77 & $\ldots$ & 0 \\
\hline \hline
\end{tabular}

Factorizable and nonfactorizable amplitudes, branching fractions and decay asymmetries of all the two-body weak decays of $\Omega_{c}$, including CF, SCS, and DCS processes, are summarized in Table IV. The channel $\Omega_{c} \rightarrow \Xi^{0} \bar{K}^{0}$ is the unique $\mathrm{CF}$ mode among all the $\Omega_{c} \rightarrow \mathcal{B} P$ decays, where $\mathcal{B}$ is a baryon octet. In both $S$ - and $P$-wave amplitudes, the nonfactorizable contributions are large and give destructive interference between factorizable ones. The branching fraction with full factorizable and nonfactorizable contributions is predicted to be $3.78 \%$. The benchmark channel $\Omega_{c} \rightarrow \Omega^{-} \pi^{+}$, which is also classified into CF modes, proceeds through external $W$ emission and hence, receives only factorizable contribution. Naively, if the nonfactorizable terms of $\Omega_{c} \rightarrow \Xi^{0} \bar{K}^{0}$ are turned off, the predicted value of its branching fraction would be $2.44 \%$. This partially helps to understand the Belle measurement of large relative ratio between $\Omega_{c} \rightarrow \Xi^{0} \bar{K}^{0}$ and $\Omega_{c} \rightarrow \Omega^{-} \pi^{+}$; see Eq. (3). A detailed consideration for these channels decaying into baryon decuplet will be presented in a separate work. ${ }^{3}$ Although no explicit measurement of the mode $\Omega_{c} \rightarrow$ $\Xi^{0} \bar{K}^{0}$ has been given, a large branching fraction prediction indicates a direct measurement is promising in the near future. The decay asymmetry $\alpha$ is predicted to be positive and with a measurable value 0.51 , which is also testable when more data are available.

The three decay modes $\Omega_{c} \rightarrow \Sigma^{+} K^{-}, \Sigma^{0} \bar{K}^{0}, \Lambda^{0} \bar{K}^{0}$ in SCS channels, which do not receive factorizable contributions, are typical examples for the essential role of nonfactorizable contribution in charmed baryon decays. Due to the breaking SU(3) flavor symmetry, see parameter $X_{1}^{s}$, the

\footnotetext{
${ }^{3}$ A prediction for branching fraction of $\Omega_{c} \rightarrow \Omega^{-} \pi^{+}$, based on an early work in [19] with updated $a_{1}=1.26$ and latest $\Omega_{c}$ lifetime, is of order $9 \%$. The incompatibility among our prediction of $\Omega_{c} \rightarrow \Xi^{0} \bar{K}^{0}$, the prediction of $\Omega_{c} \rightarrow \Omega^{-} \pi^{+}$in [19], and Belle measurement Eq. (3) will also be discussed therein.
}

$S$-wave amplitudes for the modes with $\Sigma$ baryon final states are tiny but not vanishing. On the other hand, $S$-wave amplitude for $\Omega_{c} \rightarrow \Lambda^{0} \bar{K}^{0}$ is significantly enhanced from $\Sigma^{0} \bar{K}^{0}$ for its typical size is described by $X_{2}^{s}$, which is 2 orders of magnitude larger than $X_{1}^{s}$. Among the $P$-wave terms of the three modes, $\Sigma^{+} K^{-}$is the largest one as cancellation occurs in the other two modes. Since $S$-wave amplitude dominates the branching fraction, according to Eq. (5), $\Omega_{c} \rightarrow \Lambda^{0} \bar{K}^{0}$ is predicted with the largest branching fraction among the three modes. However, the decay asymmetries for all the three modes is tiny, which is a natural consequence of tiny value for either $A$ or $B$. The remaining two modes in SCS processes, $\Omega_{c} \rightarrow \Xi^{0} \pi^{0}$ and $\Xi^{-} \pi^{+}$, share almost the same factorizable contributions, while in both channels, the nonfactorizable terms contribute constructively in $S$-wave and destructively in $P$-wave terms, leading to tiny decay asymmetries again.

Predictions for DCS channels are also summarized in Table IV. The mode $\Omega_{c} \rightarrow \Lambda^{0} \eta$ is of particular interest in all the DCS channels. Its $S$ - and $P$-wave amplitudes, which both are depicted by $X_{2}^{D}$, are substantial and hence, lead to a large branching fraction $0.36 \%$. The large and positive decay asymmetry 0.66 is also predicted. The $S$-wave amplitudes for the channels $\Omega_{c} \rightarrow \Sigma \pi / \eta$ and $\Omega_{c} \rightarrow \Lambda \pi^{0}$ vanish among all the modes which contain net nonfactorizable contribution due to the reasons $I_{3}\left(\Sigma^{0}\right)=I_{3}(\Lambda)=0$ or $X_{1}^{D}=0$. Then, the two decays $\Omega_{c} \rightarrow \Sigma^{0} \eta$ and $\Lambda^{0} \pi^{0}$ are prohibited due to the properties $g_{\Lambda^{0} \Lambda^{0}}^{A\left(\pi^{0}\right)}=g_{\Sigma^{0} \Lambda^{0}}^{A\left(\eta_{8}\right)}=0$, furthermore. The identical predictions for the three $\Sigma \pi$ modes are natural consequences in the pole model. The vanishing $S$-wave amplitudes of the three modes leads to their null

\footnotetext{
${ }^{4}$ The two DCS channels $\Omega_{c} \rightarrow \Sigma^{0} \eta$ and $\Lambda^{0} \pi^{0}$ are not included in the table for their vanishing $S$-wave, tiny $P$-wave amplitudes, and hence almost zero branching fractions.
} 
TABLE V. Predicted branching fractions in the unit of $10^{-2}, 10^{-3}$, and $10^{-4}$ (upper entry in each mode) and decay asymmetry $\alpha$ (lower entry) of $\Omega_{c}$ decays by different groups.

\begin{tabular}{|c|c|c|c|c|c|c|}
\hline Mode & Our & Cheng et al. CA [20] & Cheng et al. pole model [20] & Körner et al. [18] & Ivanov et al. [21] & Zhao [23] \\
\hline$\Omega_{c} \rightarrow \Xi^{0} \bar{K}^{0}$ & $\begin{array}{l}3.78 \\
0.51\end{array}$ & $\begin{array}{l}2.63 \\
0.44\end{array}$ & $\begin{array}{c}0.35 \\
-0.93\end{array}$ & $\begin{array}{l}4.69 \\
0.51\end{array}$ & $\begin{array}{c}0.09 \\
-0.81\end{array}$ & \\
\hline$\Omega_{c} \rightarrow \Xi^{-} \pi^{+}$ & $\begin{array}{c}9.34 \\
-0.03\end{array}$ & & & & & 0.7 \\
\hline$\Omega_{c} \rightarrow \Xi^{-} K^{+}$ & $\begin{array}{l}9.58 \\
0.27\end{array}$ & & & & & 0.6 \\
\hline
\end{tabular}

decay asymmetries, while the identical branching fractions are caused by their similar pole diagrams associated with the isospin factor $1 / \sqrt{2}$.

\section{B. Comparison with other works}

In the early 1990s, there were many efforts to study charmed baryon decays, among which few were $\Omega_{c}$ involved [18-21]. Later, semileptonic decays of heavy $\Omega$ baryons, including $\Omega_{c}$, was studied in [23,38]. In recent years, there have been some interests on its hadronic weak decays [22,23,39], in which [22] focused on modes with axial-vector final state, and only modes with decuplet baryon final state were partially involved in [39]. In Table V, a comparison with other groups, whose predictions have been updated by incorporating current $\Omega_{c}$ lifetime, is summarized in available channels.

The CF channel $\Omega_{c} \rightarrow \Xi^{0} \bar{K}^{0}$ attracts more attentions in the past $[18,20,21]$, and nonfactorizable contributions have been incorporated by all groups. Based on the pole mode combing current algebra, our results both for branching fraction and decay asymmetry can be confirmed by the early calculation within the same approach [20]. However, the prediction for the branching fraction is around 10 times larger than an early estimation that relied on a pure pole model [20], and the sign of decay asymmetry is opposite. Small branching fraction and negative asymmetry were also predicted within a relativistic three-quark model with a Gaussian shape for the momentum dependence of a baryon-three-quark vertex [21]. Such a situation occurred in the studies on antitriplet charmed baryons [27,28]. Taking the mode $\Lambda_{c} \rightarrow \Sigma^{+} \pi^{0}$ as an example, the pure pole model in [20] and quark model calculation in [21] both predicted positive decay asymmetry, while current algebra predicted $\alpha=-0.76$, which is consistent with experimental value $\alpha=-0.55 \pm 0.11$ [1]. Interestingly, working in an independent approach, Körner-Krämer gave a consistent prediction for both the branching fraction and decay asymmetry in covariant quark model [18].

The branching fractions of the SCS process $\Omega_{c} \rightarrow \Xi^{-} \pi^{+}$ and DCS process $\Omega_{c} \rightarrow \Xi^{-} K^{+}$were estimated in [23], where baryon-baryon transition form factors were calculated in the light-front quark model and only the factorizable contribution was taken into account. It has been widely accepted that nonfactorizable contribution should play an essential role in the hadronic decays. The numerical results for each individual term in Table IV show that nonfactorizable terms even give dominated contributions, which helps to explain why our prediction is more than 10 times larger.

\section{CONCLUSIONS}

In this work, we have systematically studied the branching fractions and up-down decay asymmetries of CF, SCS, and DCS decays of $\Omega_{c}$, the heaviest singly charmed baryon which decays weakly. Both factorizable and nonfactorizable terms have been taken into account in the calculation of $S$ - and $P$-wave amplitudes. To estimate the nonfactorizable contribution, we work in the pole model for $P$-wave amplitudes and current algebra for $S$-wave ones. All the nonperturbative parameters, including baryon-baryon transition form factors, baryon matrix elements, and axialvector form factors, are evaluated within MIT bag model throughout the whole calculations.

Some conclusions can be drawn from our analysis as follows:

(i) The channel $\Omega_{c} \rightarrow \Xi^{0} \bar{K}^{0}$ is the unique mode for $\mathrm{CF}$ decay. Although no absolute branching fraction has been measured up to now, the predicted large value for branching fraction indicates this mode is quite promising to be measured in the near future. Meanwhile, its decay asymmetry is predicted to be large in magnitude and positive in sign.

(ii) Among all the SCS modes, the channel $\Omega_{c} \rightarrow \Lambda^{0} \bar{K}^{0}$ is special as it proceeds only through the nonfactorizable contributions. Though the $P$-wave amplitude is small, its large $S$-wave amplitude leads to a large branching ratio. Hence, a measurement of $\Omega_{c} \rightarrow$ $\Lambda^{0} \bar{K}^{0}$ in the future will demonstrate the essential role of nonfactorizable contribution in charmed baryon weak decays.

(iii) The decay asymmetries of all SCS modes are small, which is a natural consequence of either small $S$ - or $P$-wave amplitude. In other words, it is difficult to measure decay asymmetries of SCS $\Omega_{c}$ weak decay in experiment.

(iv) The measurement of $\Omega_{c} \rightarrow \Lambda \eta$ will also be interesting. Although this mode is classified as the DCS 
mode, not only the branching fraction is predicted to be large, but also its decay asymmetry is predicted to be large in magnitude and positive in sign, which makes the measurement conceivable in experiment. Such features can be explained by simultaneous large $S$ - and $P$-wave amplitudes.

(v) The two DCS modes $\Omega_{c} \rightarrow \Sigma^{0} \eta$ and $\Omega_{c} \rightarrow \Lambda^{0} \pi^{0}$ are forbidden, for both $S$ - and $P$-wave amplitudes are found to be zero. On the other hand, these two modes can serve as golden channels for new physics searching.

\section{ACKNOWLEDGMENTS}

We would like to thank Professor Hai-Yang Cheng for his encouragement and fruitful discussion of this work. This research is supported by NSFC under Grants No. U1932104 and No. 11605076.

Note added.-S. H. and G. M. contribute equally and are co-first authors, while F.X. is corresponding author.

\section{APPENDIX A: COMMUTATORS IN $S$-WAVE AMPLITUDES}

The nonfactorizable $S$-wave amplitude is determined by the commutator terms of conserving charge $Q^{a}$ and the parity-conserving part of the Hamiltonian, shown in Eq. (22). In terms of such commutators, we further present the $A^{\text {com }}$ for various meson production more explicitly,

$$
\begin{aligned}
& A^{\mathrm{com}}\left(B_{i} \rightarrow B_{f} \pi^{ \pm}\right)=\frac{1}{f_{\pi}}\left\langle B_{f}\left|\left[I_{\mp}, H_{\mathrm{eff}}^{P C}\right]\right| B_{i}\right\rangle, \\
& A^{\mathrm{com}}\left(B_{i} \rightarrow B_{f} \pi^{0}\right)=\frac{\sqrt{2}}{f_{\pi}}\left\langle B_{f}\left|\left[I_{3}, H_{\mathrm{eff}}^{P C}\right]\right| B_{i}\right\rangle, \\
& A^{\operatorname{com}}\left(B_{i} \rightarrow B_{f} \eta_{8}\right)=\sqrt{\frac{3}{2}} \frac{1}{f_{\eta_{8}}}\left\langle B_{f}\left|\left[Y, H_{\mathrm{eff}}^{P C}\right]\right| B_{i}\right\rangle, \\
& A^{\mathrm{com}}\left(B_{i} \rightarrow B_{f} K^{ \pm}\right)=\frac{1}{f_{K}}\left\langle B_{f}\left|\left[V_{\mp}, H_{\mathrm{eff}}^{P C}\right]\right| B_{i}\right\rangle, \\
& A^{\mathrm{com}}\left(B_{i} \rightarrow B_{f} \bar{K}^{0}\right)=\frac{1}{f_{K}}\left\langle B_{f}\left|\left[U_{+}, H_{\mathrm{eff}}^{P C}\right]\right| B_{i}\right\rangle, \\
& A^{\mathrm{com}}\left(B_{i} \rightarrow B_{f} K^{0}\right)=\frac{1}{f_{K}}\left\langle B_{f}\left|\left[U_{-}, H_{\mathrm{eff}}^{P C}\right]\right| B_{i}\right\rangle .
\end{aligned}
$$

In Eq. (A3), $\eta_{8}$ is the octet component of the $\eta$ and $\eta^{\prime}$,

$$
\eta=\cos \theta \eta_{8}-\sin \theta \eta_{0}, \quad \eta^{\prime}=\sin \theta \eta_{8}+\cos \theta \eta_{0},
$$

with $\theta=-15.4^{\circ}$ [40]. For the decay constant $f_{\eta_{8}}$, we shall follow [40] to use $f_{\eta_{8}}=f_{8} \cos \theta$ with $f_{8}=1.26 f_{\pi}$. The convention for the hypercharge $Y$ is taken to be $Y=B+S-C[27]$.

\section{APPENDIX B: HADRONIC MATRIX ELEMENTS}

The baryonic matrix elements $a_{\mathcal{B}^{\prime} \mathcal{B}}$ get involved both in $S$-wave and $P$-wave amplitudes. Their general expression in terms of the effective Hamiltonian Eq. (7) is given by

$$
\begin{aligned}
a_{\mathcal{B}^{\prime} \mathcal{B}} \equiv & \left\langle\mathcal{B}^{\prime}\left|\mathcal{H}_{\mathrm{eff}}^{\mathrm{PC}}\right| \mathcal{B}\right\rangle \\
= & \begin{cases}\frac{G_{F}}{2 \sqrt{2}} V_{c s} V_{u d^{*}}^{*} c_{-}\left\langle\mathcal{B}^{\prime}\left|O_{-}\right| \mathcal{B}\right\rangle, & \mathrm{CF} \\
\frac{G_{F}}{2 \sqrt{2}} \sum_{q} V_{c q} V_{u q}^{*} c_{-}\left\langle\mathcal{B}^{\prime}\left|O_{-}^{q}\right| \mathcal{B}\right\rangle, & \mathrm{SCS} \\
\frac{G_{F}}{2 \sqrt{2}} V_{c d} V_{u s}^{*} c_{-}\left\langle\mathcal{B}^{\prime}\left|O_{-}^{D}\right| \mathcal{B}\right\rangle, & \mathrm{DCS},\end{cases}
\end{aligned}
$$

where $\quad O_{ \pm}=(\bar{s} c)(\bar{u} d) \pm(\bar{s} d)(\bar{u} c), \quad O_{ \pm}^{q}=(\bar{q} c)(\bar{u} q) \pm$ $(\bar{q} q)(\bar{u} c)$ (with $q=d, s)$, and $O_{ \pm}^{D}=(\bar{d} c)(\bar{u} s) \pm(\bar{d} s)(\bar{u} c)$ and $c_{ \pm}=c_{1} \pm c_{2}$. The matrix element of $O_{+}^{(q, D)}$ vanishes as this operator is symmetric in color indices. We shall calculate relevant baryon matrix elements in MIT bag model. The results for $\mathrm{CF}$ processes are

$$
\begin{aligned}
& \left\langle\Xi^{0}\left|O_{-}\right| \Xi_{c}^{\prime 0}\right\rangle=-\frac{2 \sqrt{2}}{3}\left(X_{1}+9 X_{2}\right)(4 \pi), \\
& \left\langle\Xi^{0}\left|O_{-}\right| \Xi_{c}^{0}\right\rangle=\frac{2 \sqrt{6}}{3}\left(X_{1}-3 X_{2}\right)(4 \pi) .
\end{aligned}
$$

Likewise, the matrix elements for SCS decays are calculated to be

$$
\begin{aligned}
\left\langle\Xi^{0}\left|O_{-}^{d}\right| \Omega_{c}^{0}\right\rangle & =0, \quad\left\langle\Xi^{0}\left|O_{-}^{s}\right| \Omega_{c}^{0}\right\rangle=-\frac{4}{3}\left(X_{1}^{s}+9 X_{2}^{s}\right)(4 \pi), \\
\left\langle\Sigma^{+}\left|O_{-}^{d}\right| \Xi_{c}^{\prime+}\right\rangle & =0, \quad\left\langle\Sigma^{+}\left|O_{-}^{s}\right| \Xi_{c}^{\prime+}\right\rangle=\frac{2 \sqrt{2}}{3}\left(X_{1}^{s}-9 X_{2}^{s}\right)(4 \pi), \\
\left\langle\Sigma^{0}\left|O_{-}^{d}\right| \Xi_{c}^{\prime 0}\right\rangle & =\frac{4}{3} X_{1}^{d}(4 \pi), \\
\left\langle\Sigma^{0}\left|O_{-}^{s}\right| \Xi_{c}^{\prime 0}\right\rangle & =-\frac{2}{3}\left(X_{1}^{s}-9 X_{2}^{s}\right)(4 \pi) \\
\left\langle\Lambda^{0}\left|O_{-}^{d}\right| \Xi_{c}^{\prime 0}\right\rangle & =-4 \sqrt{3} X_{2}^{d}(4 \pi) \\
\left\langle\Lambda^{0}\left|O_{-}^{s}\right| \Xi_{c}^{\prime 0}\right\rangle & =-\frac{2 \sqrt{3}}{3}\left(X_{1}^{s}+3 X_{2}^{s}\right)(4 \pi) \\
\left\langle\Sigma^{0}\left|O_{-}^{d}\right| \Xi_{c}^{0}\right\rangle & =-\frac{4 \sqrt{3}}{3} X_{1}^{d}(4 \pi) \\
\left\langle\Sigma^{0}\left|O_{-}^{s}\right| \Xi_{c}^{0}\right\rangle & =-\frac{2 \sqrt{3}}{3}\left(X_{1}^{s}+3 X_{2}^{s}\right)(4 \pi) \\
\left\langle\Lambda^{0}\left|O_{-}^{d}\right| \Xi_{c}^{0}\right\rangle & =-4 X_{2}^{d}(4 \pi) \\
\left\langle\Lambda^{0}\left|O_{-}^{s}\right| \Xi_{c}^{0}\right\rangle & =-2\left(X_{1}^{s}-X_{2}^{s}\right)(4 \pi),
\end{aligned}
$$

and for DCS processes are

$$
\begin{aligned}
\left\langle\Sigma^{0}\left|O_{-}^{D}\right| \Omega_{c}^{0}\right\rangle & =\frac{4}{3} \sqrt{2} X_{1}^{D}(4 \pi), \\
\left\langle\Lambda\left|O_{-}^{D}\right| \Omega_{c}^{0}\right\rangle & =-4 \sqrt{6} X_{2}^{D}(4 \pi),
\end{aligned}
$$


where we have introduced the bag integrals $X_{1}$ and $X_{2}$,

$$
\begin{array}{lll}
X_{1}=\int_{0}^{R} r^{2} d r\left(u_{s} v_{u}-v_{s} u_{u}\right)\left(u_{c} v_{d}-v_{c} u_{d}\right), & X_{2}=\int_{0}^{R} r^{2} d r\left(u_{s} u_{u}+v_{s} v_{u}\right)\left(u_{c} u_{d}+v_{c} v_{d}\right), \\
X_{1}^{d}=\int_{0}^{R} r^{2} d r\left(u_{u} v_{u}-v_{u} u_{u}\right)\left(u_{c} v_{u}-v_{c} u_{u}\right), & X_{2}^{d}=\int_{0}^{R} r^{2} d r\left(u_{u} u_{c}+v_{u} v_{c}\right)\left(u_{u} u_{u}+v_{u} v_{u}\right), \\
X_{1}^{s}=\int_{0}^{R} r^{2} d r\left(u_{s} v_{u}-v_{s} u_{u}\right)\left(u_{c} v_{s}-v_{c} u_{s}\right), & X_{2}^{s}=\int_{0}^{R} r^{2} d r\left(u_{s} u_{u}+v_{s} v_{u}\right)\left(u_{c} u_{s}+v_{c} v_{s}\right), \\
X_{1}^{D}=\int_{0}^{R} r^{2} d r\left(u_{u} v_{u}-v_{u} u_{u}\right)\left(u_{c} v_{s}-v_{c} u_{s}\right), & X_{2}^{D}=\int_{0}^{R} r^{2} d r\left(u_{u} u_{u}+v_{u} v_{u}\right)\left(u_{c} u_{s}+v_{c} v_{s}\right),
\end{array}
$$

with the numbers $X_{1}=3.56 \times 10^{-6}, X_{2}=1.74 \times 10^{-4}, X_{1}^{d}=0, X_{2}^{d}=1.60 \times 10^{-4}, X_{1}^{s}=2.60 \times 10^{-6}, X_{2}^{s}=1.96 \times 10^{-4}$, $X_{1}^{D}=0, X_{2}^{D}=1.78 \times 10^{-4}$. To obtain numerical results, we have employed the following bag parameters:

$$
m_{u}=m_{d}=0, \quad m_{s}=0.279 \mathrm{GeV}, \quad m_{c}=1.551 \mathrm{GeV}, \quad R=5 \mathrm{GeV}^{-1},
$$

where $R$ is the radius of the bag.

\section{APPENDIX C: AXIAL-VECTOR FORM FACTORS}

The axial-vector form factor in the static limit can be expressed in the bag model as

$$
g_{\mathcal{B}^{\prime} \mathcal{B}}^{A(P)}=\left\langle\mathcal{B}^{\prime} \uparrow\left|b_{q_{1}}^{\dagger} b_{q_{2}} \sigma_{z}\right| \mathcal{B} \uparrow\right\rangle \int d^{3} \boldsymbol{r}\left(u_{q_{1}} u_{q_{2}}-\frac{1}{3} v_{q_{1}} v_{q_{2}}\right) .
$$

The relevant results are

$$
\begin{aligned}
& g_{\Xi_{c}^{0} \Omega_{c}^{0}}^{A\left(\bar{K}^{0}\right)}=-\frac{\sqrt{6}}{3}\left(4 \pi Z_{2}\right), \quad g_{\Xi_{c}^{\prime 0} \Omega_{c}^{0}}^{A\left(\bar{K}^{0}\right)}=\frac{2 \sqrt{2}}{3}\left(4 \pi Z_{2}\right), \quad g_{\Xi^{-} \Xi^{0}}^{A\left(\pi^{+}\right)}=-\frac{1}{3}\left(4 \pi Z_{1}\right), \\
& g_{\Sigma^{+} \Xi^{0}}^{A\left(K^{-}\right)}=\frac{5}{3}\left(4 \pi Z_{2}\right), \quad g_{\Omega_{c}^{0} \Xi^{0}}^{A\left(\bar{K}^{0}\right)}=0, \quad g_{\Xi^{0} \Xi^{0}}^{A\left(\pi^{0}\right)}=-\frac{1}{6}\left(4 \pi Z_{1}\right), \\
& g_{\Xi^{0} \Lambda^{0}}^{A\left(K^{0}\right)}=\frac{\sqrt{6}}{6}\left(4 \pi Z_{2}\right), \quad g_{\Xi^{0} \Sigma^{0}}^{A\left(K^{0}\right)}=-\frac{5 \sqrt{2}}{6}\left(4 \pi Z_{2}\right), \quad g_{\Omega_{c}^{0} \Omega_{c}^{0}}^{A\left(\eta_{8}\right)}=-\frac{4 \sqrt{6}}{9}\left(4 \pi Z_{1}\right), \\
& g_{\Sigma^{0} \Sigma^{0}}^{A\left(\eta_{8}\right)}=\frac{\sqrt{6}}{3}\left(4 \pi Z_{1}\right), \quad g_{\Sigma^{0} \Lambda^{0}}^{A\left(\eta_{8}\right)}=0, \quad g_{\Lambda^{0} \Sigma^{0}}^{A\left(\eta_{8}\right)}=0, \\
& g_{\Lambda^{0} \Lambda^{0}}^{A\left(\eta_{8}\right)}=-\frac{\sqrt{6}}{3}\left(4 \pi Z_{1}\right), \quad g_{\Sigma^{-} \Sigma^{0}}^{A\left(\pi^{+}\right)}=\frac{2 \sqrt{2}}{3}\left(4 \pi Z_{1}\right), \quad g_{\Sigma^{-} \Lambda^{0}}^{A\left(\pi^{+}\right)}=\frac{\sqrt{6}}{3}\left(4 \pi Z_{1}\right), \\
& g_{\Xi^{-} \Sigma^{0}}^{A\left(K^{+}\right)}=-\frac{5 \sqrt{2}}{6}\left(4 \pi Z_{2}\right), \quad g_{\Xi^{-} \Lambda^{0}}^{A\left(K^{+}\right)}=-\frac{\sqrt{6}}{6}\left(4 \pi Z_{2}\right), \quad g_{\Sigma^{+} \Sigma^{0}}^{A\left(\pi^{-}\right)}=-\frac{2 \sqrt{2}}{3}\left(4 \pi Z_{1}\right), \\
& g_{\Sigma^{+} \Lambda^{0}}^{A\left(\pi^{-}\right)}=\frac{\sqrt{6}}{3}\left(4 \pi Z_{1}\right), \quad g_{\Sigma^{0} \Lambda^{0}}^{A\left(\pi^{0}\right)}=\frac{\sqrt{3}}{3}\left(4 \pi Z_{1}\right), \quad g_{\Sigma^{0} \Sigma^{0}}^{A\left(\pi^{0}\right)}=0, \\
& g_{\Lambda^{0} \Lambda^{0}}^{A\left(\pi^{0}\right)}=0, \quad g_{\Lambda^{0} \Sigma^{0}}^{A\left(\pi^{0}\right)}=\frac{\sqrt{3}}{3}\left(4 \pi Z_{1}\right), \quad g_{\Sigma^{0} \Xi^{0}}^{A\left(\bar{K}^{0}\right)}=-\frac{5 \sqrt{2}}{6}\left(4 \pi Z_{2}\right), \\
& g_{\Lambda^{0} \Xi^{0}}^{A\left(\bar{K}^{0}\right)}=\frac{\sqrt{6}}{6}\left(4 \pi Z_{2}\right), \quad g_{\Omega^{-} \Xi^{0}}^{A\left(K^{+}\right)}=-\frac{2 \sqrt{6}}{3}\left(4 \pi Z_{2}\right),
\end{aligned}
$$

where the auxiliary bag integrals are given by

$$
Z_{1}=\int r^{2} d r\left(u_{u}^{2}-\frac{1}{3} v_{u}^{2}\right), \quad Z_{2}=\int r^{2} d r\left(u_{u} u_{s}-\frac{1}{3} v_{u} v_{s}\right)
$$

Numerically, $(4 \pi) Z_{1}=0.65$ and $(4 \pi) Z_{2}=0.71$. 
[1] M. Tanabashi et al. (Particle Data Group), Review of Particle Physics, Phys. Rev. D 98, 030001 (2018).

[2] E. Solovieva et al., Study of $\Omega_{c}^{0}$ and $\Omega_{c}^{* 0}$ baryons at Belle, Phys. Lett. B 672, 1 (2009).

[3] D. Cronin-Hennessy et al. (CLEO Collaboration), Observation of the $\Omega_{c}^{0}$ Charmed Baryon at CLEO, Phys. Rev. Lett. 86, 3730 (2001).

[4] P. L. Frabetti et al. (E687 Collaboration), Observation and mass measurement of $\Omega_{c}^{0} \rightarrow \Sigma^{+} K^{-} K^{-} \pi^{+}$, Phys. Lett. B 338, 106 (1994).

[5] R. Aaij et al. (LHCb Collaboration), Observation of Five New Narrow $\Omega_{c}^{0}$ States Decaying to $\Sigma_{c}^{+} K^{-}$, Phys. Rev. Lett. 118, 182001 (2017).

[6] J. Yelton et al. (Belle Collaboration), Observation of excited $\Omega_{c}$ charmed baryons in $e^{+} e^{-}$collisions, Phys. Rev. D 97, 051102(R) (2018).

[7] H. Y. Cheng and C. W. Chiang, Quantum numbers of $\Omega_{c}$ states and other charmed baryons, Phys. Rev. D 95, 094018 (2017).

[8] S. S. Agaev, K. Azizi, and H. Sundu, On the nature of the newly discovered $\Omega$ states, Europhys. Lett. 118, 61001 (2017).

[9] H. Huang, J. Ping, and F. Wang, Investigating the excited $\Omega_{c}^{0}$ states through $\Xi_{c} \bar{K}$ and $\Xi_{c}^{\prime} \bar{K}$ decay channels, Phys. Rev. D 97, 034027 (2018).

[10] C. S. An and H. Chen, Observed $\Omega_{c}^{0}$ resonances as pentaquark states, Phys. Rev. D 96, 034012 (2017).

[11] R. Aaij et al. (LHCb Collaboration), Measurement of the $\Omega_{c}^{0}$ Baryon Lifetime, Phys. Rev. Lett. 121, 092003 (2018).

[12] J. M. Link et al. (FOCUS Collaboration), Measurement of the $\Omega_{c}^{0}$ lifetime, Phys. Lett. B 561, 41 (2003).

[13] M. I. Adamivich et al. (WA89 Collaboration), Measurement of the $\Omega_{c}^{0}$ lifetime, Phys. Lett. B 358, 151 (1995).

[14] P. L. Frabetti et al. (E687 Collaboration), First measurement of the lifetime of the $\Omega_{c}^{0}$, Phys. Lett. B 357, 678 (1995).

[15] H. Y. Cheng, Phenomenological study of heavy hadron lifetimes, J. High Energy Phys. 11 (2018) 014.

[16] J. Yelton et al. (Belle Collaboration), Measurement of branching fractions of hadronic decays of the $\Omega_{c}^{0}$ baryon, Phys. Rev. D 97, 032001 (2018).

[17] R. Ammar et al. (CLEO Collaboration), Observation of the Decay $\Omega_{c}^{0} \rightarrow \Omega^{-} e^{+} \nu_{e}$, Phys. Rev. Lett. 89, 171803 (2002).

[18] J. G. Korner and M. Kramer, Exclusive non-leptonic charm baryon decays, Z. Phys. C 55, 659 (1992).

[19] Q. P. Xu and A. N. Kamal, Nonleptonic charmed-baryon decays: $\quad B_{c} \rightarrow B\left(\frac{3}{2}^{+}\right.$, decuplet $)+P\left(0^{-}\right)$or $V\left(1^{-}\right)$, Phys. Rev. D 46, 3836 (1992).

[20] H. Y. Cheng and B. Tseng, Cabibbo-allowed nonleptonic weak decays of charmed baryons, Phys. Rev. D 48, 4188 (1993).

[21] M. A. Ivanov, J. G. Korner, V. E. Lyubovitskij, and A. G. Rusetsky, Exclusive nonleptonic decays of bottom and charm baryons in a relativistic three-quark model: Evaluation of nonfactorizing diagrams, Phys. Rev. D 57, 5632 (1998).

[22] R. Dhir and C. S. Kim, Axial-vector emitting weak nonleptonic decays of $\Omega_{c}^{0}$ baryon, Phys. Rev. D 91, 114008 (2015).
[23] Z. X. Zhao, Weak decays of heavy baryons in the light-front approach, Chin. Phys. C 42, 093101 (2018).

[24] H. Y. Cheng and B. Tseng, Nonleptonic weak decays of charmed baryons, Phys. Rev. D 46, 1042 (1992); 55, 1697(E) (1997).

[25] L. S. Brown and C. M. Sommerfield, Algebra of Currents and Nonleptonic Hyperon Decay, Phys. Rev. Lett. 16, 751 (1966).

[26] M. Gronau, Nonleptonic Hyperon Decays in a CurrentCurrent Quark Model, Phys. Rev. D 5, 118 (1972); 5, 1877(E) (1972).

[27] H. Y. Cheng, X. W. Kang, and F. Xu, Singly Cabibbosuppressed hadronic decays of $\Lambda_{c}^{+}$, Phys. Rev. D 97, 074028 (2018).

[28] J. Zou, F. Xu, G. Meng, and H. Y. Cheng, Two-body hadronic weak decays of antitriplet charmed baryons, Phys. Rev. D 101, 014011 (2020).

[29] M. Ablikim et al. (BESIII Collaboration), Measurements of weak decay asymmetries of $\Lambda_{c}^{+} \rightarrow p K_{s}^{0}, \Lambda \pi^{+}, \Sigma^{+} \pi^{0}$, and $\Sigma^{0} \pi^{+}$, Phys. Rev. D 100, 072004 (2019).

[30] H. Y. Cheng, G. Meng, F. Xu, and J. Zou, Two-body weak decays of doubly charmed baryons, Phys. Rev. D 101, 034034 (2020).

[31] L. L. Chau, H. Y. Cheng, and B. Tseng, Analysis of twobody decays of charmed baryons using the quark-diagram scheme, Phys. Rev. D 54, 2132 (1996).

[32] J. G. Korner, G. Kramer, and J. Willrodt, Weak decays of charmed baryons, Z. Phys. C 2, 117 (1979).

[33] P. Żenczykowski, Nonleptonic charmed-baryon decays: Symmetry properties of parity-violating amplitudes, Phys. Rev. D 50, 5787 (1994).

[34] G. Buchalla, A. J. Buras, and M. E. Lautenbacher, Weak decays beyond leading logarithms, Rev. Mod. Phys. 68, 1125 (1996).

[35] R. Perez-Marcial, R. Huerta, A. Garcia, and M. Avila-Aoki, Predictions for semileptonic decays of charm baryons. II. Nonrelativistic and MIT bag quark models, Phys. Rev. D 40, 2955 (1989); 44, 2203(E) (1991).

[36] H. Cheng and B. Tseng, 1/M corrections to baryonic form factors in the quark model, Phys. Rev. D 53, 1457 (1996).

[37] A. Chodos, R. L. Jaffe, K. Johnson, and C. B. Thorn, Baryon structure in the bag theory, Phys. Rev. D 10, 2599 (1974); T. A. DeGrand, R. L. Jaffe, K. Johnson, and J.E. Kiskis, Masses and other parameters of the light hadrons, Phys. Rev. D 12, 2060 (1975).

[38] M. Pervin, W. Roberts, and S. Capstick, Semileptonic decays of heavy $\Omega$ baryons in a quark model, Phys. Rev. C 74, 025205 (2006).

[39] T. Gutsche, M. A. Ivanov, J. G. Krner, and V. E. Lyubovitskij, Nonleptonic two-body decays of single heavy baryons $\Lambda_{Q}, \Xi_{Q}$, and $\Omega_{Q}(Q=b, c)$ induced by $\mathrm{W}$ emission in the covariant confined, Phys. Rev. D 98, 074011 (2018).

[40] T. Feldmann, P. Kroll, and B. Stech, Mixing and decay constants of pseudoscalar mesons: the sequel, Phys. Lett. B 449, 339 (1999); Mixing and decay constants of pseudoscalar mesons, Phys. Rev. D 58, 114006 (1998). 